\title{
PARÁMETROS HEMATOLÓGICOS DEL “GUACAMAYO CABEZA AZUL” Primolius couloni L. (PSITTACIDAE) EN CAUTIVERIO
}

\author{
Hematological P arameters of Blue-Headed Macaw Primolius couloni L. \\ (Psittacidae) in Captivity
}

\author{
Giovanna Solari $^{1,2}$, David Tribeño ${ }^{1}$, Zoila Navarro ${ }^{1}$, Rafael Alvis ${ }^{3,4}$, José Pino ${ }^{3}$
}

\section{Resumen}

\begin{abstract}
El Guacamayo de Cabeza Azul (Primolius couloni) se considera un loro del Nuevo Mundo en peligro de extinción. Para establecer rangos hematológicos de referencia, se tomaron muestras de sangre de 13 especímenes ( 7 machos, 6 hembras) criados en cautividad en el Zoológico del Patronato del Parque de Las Leyendas de Lima, Perú. El promedio de eritrocitos y leucocitos fue 2608 x $10^{3} / \mathrm{ml}$ y de 10.11 x $10^{3} / \mathrm{ml}$, respectivamente; $50.15 \%$ de hematocrito y $200 \mathrm{fl}$ de volumen corpuscular medio; asimismo, en el recuento diferencial leucocitario se observó 64.7 y $25.3 \%$ de heterófilos y leucocitos, respectivamente. El diámetro mayor y menor de los eritrocitos fue de 11.3 y $7.0 \mu \mathrm{m}$, respectivamente. Estos resultados podrían emplearse como valores locales de referencia para esta especie.
\end{abstract}

Palabras clave: guacamayo cabeza azul, Primolius couloni, Psittacidae, hematología, valores normales

\section{Abstract}

The Blue-headed macaw (Primolius couloni) is considered a New World endangered parrot. Blood samples were collected from 13 captive blue-headed macaws ( 7 male, 6 female) housed in the Las Leyendas Zoological Park in Lima, Peru to establish hematologic reference values. Mean values for erythrocytes and leukocytes were 2608 x $10^{3} / \mathrm{ml}$ and $10.11 \times 10^{3} / \mathrm{ml}$ respectively; hematocrit was $50.15 \%$ and mean corpuscular volume was 200 fl. The differential leukocyte count showed 64.7 and $25.3 \%$ of heterophils and leukocytes respectively. The largest and smallest diameter of erythrocytes was 11.3 and $7.0 \mu \mathrm{m}$ respectively. Baseline hematologic ranges were established and can be used as reference values for this species.

Key words: blue-headed sparrow, Primolius couloni, Psittacidae, hematology, normal values

\footnotetext{
${ }^{1}$ Patronato del Parque de las Leyendas Felipe Benavides Barreda, Lima

${ }^{2}$ Email: scgiovi@hotmail.com

${ }^{3}$ Laboratorio de Reproducción y Biología del Desarrollo, Facultad de Ciencias Biológicas, Universidad Nacional Mayor de San Marcos, Lima.

${ }^{4}$ Laboratorio de Biología, Universidad Científica del Sur, Lima
} 


\section{INTRODUCCIÓN}

Primolius couloni (Ara couloni, Propyrrhura couloni), pertenece a la familia de las Psittacidae y se le conoce localmente como "guacamayo cabeza azul". Su distribución en el Perú comprende los departamentos de Loreto, San Martín, Huánuco, Pasco, Junín, Cuzco, Puno, Madre de Dios y Ucayali. En Brasil, se encuentra al oeste del estado de Acre y en Bolivia al nordeste de los departamentos de Pando y la Paz (Cáceres, 2006).

Según Cáceres (2006), su tamaño promedio es de $41 \mathrm{~cm}$ y su peso varía entre 207 y 294 g. El plumaje es predominantemente verde, siendo verde amarillento en las partes ventrales. La cabeza es completamente azul con una máscara facial gris. No existe dimorfismo sexual evidente entre machos y hembras. Los adultos poseen el iris amarillo y patas rosadas, mientras los juveniles presentan iris oscuro y patas grises (Cáceres y Williams de Castro, 2006).

De acuerdo al Decreto Supremo N. ${ }^{\circ}$ 043-2004-AG aprobado el 22 de septiembre de 2004 por el Instituto Nacional de Recursos Naturales (INRENA), P. couloni se encuentra en situación vulnerable. A nivel internacional se encuentra ubicada en el Apéndice I de la Convención sobre el Comercio Internacional de Especies Amenazadas de Flora y Fauna (CITES). Por otro lado, según la Lista Roja de Especies Amenazadas de la Unión Mundial para la Naturaleza (IUCN), se encuentra en la categoría "En Peligro" (Tobias y Brightsmith, 2007).

La hematología clínica ha sido por mucho tiempo uno de los criterios más empleados como medida de diagnóstico de la salud (Tell et al., 1997; Feldman y Synk, 2006), pues, además, permite evaluar cambios resultantes de demandas fisiológicas; por ejemplo, en las aves, el número de células rojas varía según la edad, el género, hora del día, temporada del año, altitud, nutrición y nive- les circulantes de hormonas como andrógenos, estrógenos y tiroideas. Además, el estudio hematológico puede ser de gran utilidad para el conocimiento de la adaptación de las especies al medio ambiente y para el manejo de la fauna con fines de conservación de la diversidad biológica.

Tanto en uno como en otro caso, la interpretación de los resultados pasa por la determinación de la normalidad de los datos obtenidos y, en su caso, del grado de desviación que presenta un parámetro frente a lo esperado. Para ello, la importancia de disponer de datos normales o de referencia para la especie a fin de poder interpretar los valores obtenidos en un determinado individuo (Soto et al., 2009).

Existe poca información sobre $P$. couloni, tanto en vida silvestre como en cautiverio (Cáceres y Williams de Castro, 2006). En el presente estudio se establece los valores hematológicos normales de Primolius couloni (guacamayo cabeza azul) en condiciones de cautiverio.

\section{Materiales y Métodos}

El presente trabajo se realizó en las instalaciones del Patronato del Parque de las Leyendas, Lima, Perú, durante el mes de mayo de 2008. Se utilizó 13 guacamayos adultos "Cabeza azul" (Primolius couloni), de los cuales, siete eran hembras y seis machos. Las aves estaban clínicamente sanas, identificadas con un anillo de metal en el tarso, y con pesos de 250 a 400 g. Recibían una dieta balanceada de frutas, vegetales y semillas.

La captura fue mediante contención física (Aguilar et al., 2005), con una malla con mango de metal. Se colocaron en sacos de plásticos y se les pesó. Se posicionó al ave en decúbito dorsal y, con una aguja N. ${ }^{\circ} 26$, se extrajo $1.5 \mathrm{ml}$ de sangre de la vena braquial. Parte de la sangre $(1 \mathrm{ml})$ se transfirió a un tubo con heparinato de litio para el análisis 
hematológico (glóbulos rojos y blancos, hematocrito, y volumen corpuscular medio [VCM]), y con el $0.5 \mathrm{ml}$ restante se prepararon 13 frotices por individuo, doce de ellos para la observación citomorfológica y, el último, para el recuento diferencial de leucocitos.

En el recuento de glóbulos blancos y glóbulos rojos se siguió el protocolo de Natt y Herrick (1952). Para el valor del VCM se empleó la fórmula (Hematocrito, en \% x 10) / $\mathrm{N} .^{\circ}$ de eritrocitos, en millones/ml. Para el recuento diferencial de leucocitos, los frotices se colorearon con Wright ( $\mathrm{pH}$ 6.8), y se observaron con un microscopio de campo claro.

Los frotices para la observación citomorfológica se dejaron secar a temperatura ambiente, se fijaron en metanol absoluto a $8{ }^{\circ} \mathrm{C}$ por $20 \mathrm{~min}$, y se tiñeron con los colorantes Giemsa, May-Grunwald, Wright, Hematoxilina-Eosina, y Nitrato de plata. Se utilizó un ocular micrométrico incorporado al microscopio para la biometría de las células sanguíneas.
Para la determinación de los promedios y dispersión de los parámetros hematológicos, se utilizaron las medidas descriptivas, empleando la media aritmética como medida de tendencia central y la desviación estándar como medida de dispersión.

\section{Resultados y Discusión}

Los valores hematológicos promedio se muestran en el Cuadro 1. El tamaño de las células sanguíneas se indica en el Cuadro 2.

Los eritrocitos se observaron como células con morfología elíptica, cuyos extremos están ligeramente redondeados conteniendo un citoplasma acidófilo o neutro, sin granulaciones en el citoplasma. El núcleo presenta una forma redondeada, con cromatina que presenta una característica homogénea y ligeramente granular (Fig. 1a). El citoplasma es rosáceo y el núcleo es rojo.

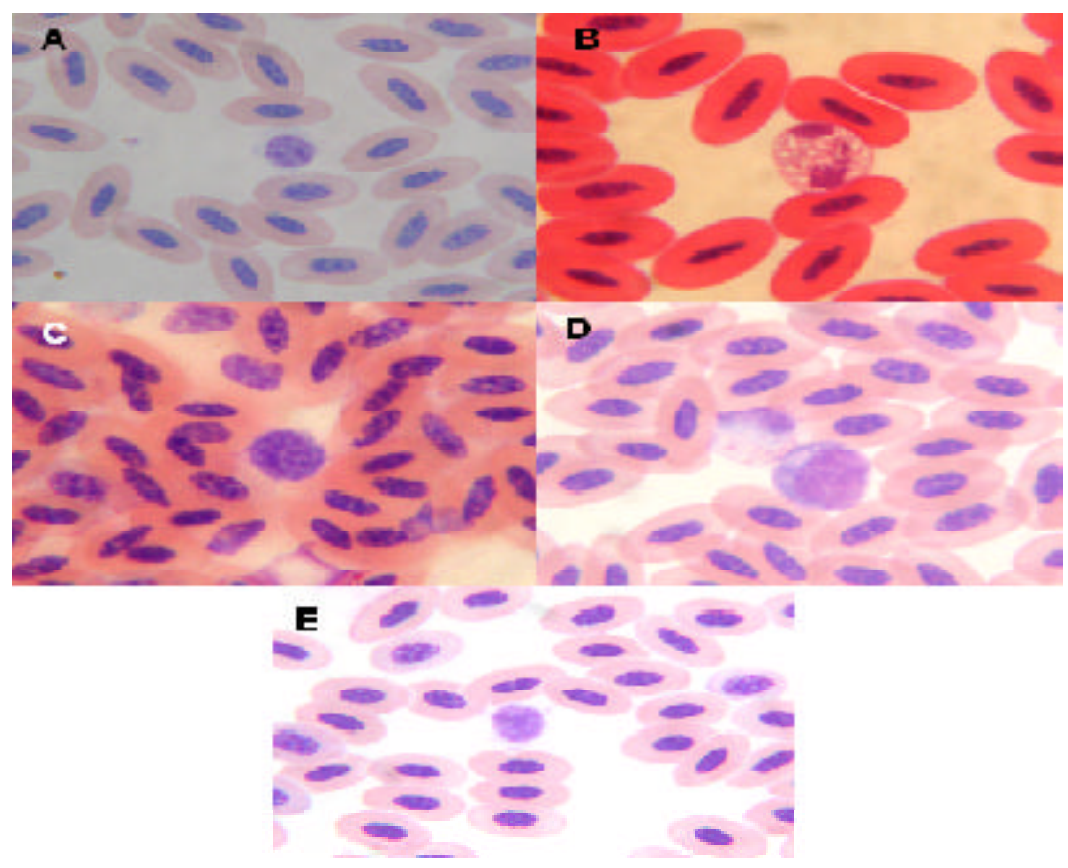

Figura 1. Morfos sanguíneos de Primolius couloni obtenidos en cautiverio. A. Eritrocitos, B. Heterófilos, C. Basófilos, D. Monocito y E. Linfocito. Coloración May-Grunwald Giemsa. Aumento: 100X 
Cuadro 1. Valores promedio de las series eritrocítica y leucocítica de 13 guacamayos adultos "Cabeza azul" (Primolius couloni), mantenidos en cautiverio en un zoológico de la ciudad de Lima

\begin{tabular}{lclc}
\hline \multicolumn{2}{c}{ Serie eritrocítica } & \multicolumn{2}{c}{ Serie leucocítica } \\
\hline Variables & Promedio + d.e. & Variables & Promedio + d.e. \\
\hline Eritrocitos $(\mathrm{x} \mathrm{10} / \mathrm{ml})$ & $2608 \pm 557$ & Leucocitos $(\mathrm{x} \mathrm{10} / \mathrm{ml})$ & $10.11 \pm 4.63$ \\
Hematocrito $(\%)$ & $50.15 \pm 4.06$ & Heterófilos $(\%)$ & $64.69 \pm 17.55$ \\
VCM (fl) & $200 \pm 36.06$ & Linfocitos $(\%)$ & $25.31 \pm 12.92$ \\
& & Monocitos (\%) & $5.69 \pm 3.75$ \\
& & Eosinófilos (\%) & $3.39 \pm 3.71$ \\
& & Basófilos $(\%)$ & $0.92 \pm 0.83$ \\
\hline
\end{tabular}

Cuadro 2. Promedio y desviación estándar del diámetro mayor y menor de células sanguíneas de 13 guacamayos adultos "Cabeza azul" (Primolius couloni), mantenidos en cautiverio en un zoológico de la ciudad de Lima

\begin{tabular}{lcc}
\hline Célula & Diámetro mayor $(\mu \mathrm{m})$ & Diámetro menor $(\mu \mathrm{m})$ \\
\hline Eritrocito & $11.3 \pm 0.79$ & $7.0 \pm 1.10$ \\
Heterófilo & $11.0 \pm 0.88$ & - \\
Linfocito & $7.3 \pm 0.71$ & - \\
Basófilo & $8.1 \pm 0.74$ & - \\
Monocito & $8.9 \pm 0.87$ & - \\
\hline
\end{tabular}

Los heterófilos son considerados "la ventana del estado de salud del ave" (McDonald, 1996), tienen forma redondeada, presentan un núcleo segmentado hasta con tres lobulaciones bien definidas, con pequeñas granulaciones violeta oscura que enmascaran al citoplasma azul celeste o rosado y finamente granulado (Fig. 1b). Las células basófilas, como en la mayoría de las células provenientes de la línea hematopoyética, poseen una forma redondeada, con un citoplasma con granulaciones más prominentes (Fig. 1c). Los monocitos presentaron un núcleo grande de forma irregular con granulaciones bien definidas, con un citoplasma compacto y homogéneo sin granulaciones (Fig. 1d).
Los linfocitos fueron, generalmente, esféricos, de tamaño diverso, presentándose también algunas células con forma irregular. Algunos poseen un núcleo más eucromático que los heterófilos, cuya característica principal es que el radio del núcleo en relación al citoplasma es alto, lo cual indica que el núcleo casi ocupa todo el citoplasma de la célula (Fig. 1e).

El tamaño de los eritrocitos, en las aves, oscila entre 11 a 16 micras para el diámetro mayor y de 6 a 10 micras para el diámetro menor (Bergman, 1957). En el caso de $P$. couloni, los valores obtenidos se encuentran dentro de los límites inferiores. 
El número de eritrocitos en las aves varía entre 1.5 a $6.6 \times 10^{6} / \mathrm{ml}$ y es inversamente proporcional al peso del ave; esto es, aves con mayor peso tienen menor cantidad de glóbulos rojos (Bergman, 1957). Por ejemplo, el colibrí rubí (Chrysolampis mosquitus) tiene un peso de 2-4 g y un promedio de 6.59 x $10^{6} / \mathrm{ml}$, en tanto que el avestruz (Struthio camelos) tiene un peso de $120 \mathrm{~kg}$ y solo 1.9 x $10^{6} / \mathrm{ml}$ (Saint-Girons y Duguy, 1963). El $P$. couloni es un ave de tamaño intermedio y presenta un promedio de $2608 \times 10^{3} / \mathrm{ml}$.

En psitácidos, los valores de hematocrito se encuentran entre 37 y $53 \%$ (Saint-Girons y Duguy, 1963), y en el caso de P. couloni fue de $50.15 \%$. Asimismo, los valores leucocitarios en la mayoría de los psitácidos varían entre 8 a $15 \times 10^{3} / \mathrm{ml}$ (Cruz et al., 2008), y en este caso fue de $10 \times 10^{3} / \mathrm{ml}$.

Mc Donald (1996) propone valores hematológicos de referencia para un grupo de psitácidos donde los datos obtenidos en el presente estudio se encuentran dentro de los rangos propuestos con excepción de los eosinófilos y monocitos. Se observan diferencias en la proporción de heterófilos y linfocitos entre las especies aviares. Por ejemplo, Cotorra cubana (Amazona leucocephala) tiene de 23 a $65 \%$ de heterófilos y de 34 a 68\% de linfocitos; Catey (Aratinga euops) de 32 a $65 \%$ de heterófilos y de 31 a $58 \%$ de linfocitos (Soto, 2010); Guacamayo de Alas Naranjas del Amazonas (Amazona amazonica amazónica) tiene $61.9 \%$ de heterófilos, y el Loro Frente Azul (Amazona aestiva) presenta un balance entre heterófilos y linfocitos (46 y 49\%, respectivamente) (Saint-Girons y Duguy,1963). Similar resultado obtiene Silveira (2006) estudiando al papagayo verdadero (A. aestiva) (49 y $48 \%$, respectivamente), mientras que $P$. couloni presentó 64.7 y $25.3 \%$ de heterófilos y linfocitos, respectivamente.

Se concluye que los valores hematológicos y morfológicos para $P$. couloni se encuentran dentro de los rangos reportados para otros géneros psitácidos. Por ser el primer reporte local en esta especie, los datos obtenidos pueden ser usados como valores referenciales para la especie.

\section{Agradecimiento}

Los autores expresan su agradecimiento a la MV Patricia Ríos por sus valiosos aportes al presente estudio.

\section{Literatura Citada}

1. Aguilar R, Hernández M, Hernández J. 2005. Atlas de medicina terapéutica y patología de animales exóticos. Argentina: Inter. Médica. 375 p.

2. Bergman RAM. 1957. The erythrocyte of snakes. Folia Haematol 75: 92-111.

3. Cáceres A, Williams de Castro M. 2006.

Porcentaje de hembras del guacamayo cabeza azul Primolius couloni (Sclater, 1876) mantenidos en cautiverio en cuatro centros de manejo de fauna silvestre en el Perú. Ecol Aplicada 5: 136-139.

4. Cruz M, Arraga-Alvarado C, Rincón M, Fernández G, Aguilar J, VillasmilOntiveros Y, Gómez O, Henríquez A. 2008. Valores hematológicos de psitácidos de los géneros Ara y Amazona cautivos en zoológicos de Venezuela. Rev Cient 18: 469-661.

5. Feldman B, Sink C. 2006. Urinálise hematologia laboratorial para o clínico de pequenos animais. São Paulo: Roca. $111 \mathrm{p}$.

6. McDonald S. 1996. The complete blood count. Avian Quarterly [Internet], [28 octubre 2010]. Disponible en: http:// www.oldworldaviaries.com/text/ miscellaneous/blood_count.html

7. Natt MP, Herrick CA. 1952. A new diluent for counting erytrhocytes and leucocytes of the chicken. Poultry Sci 31: 735-738.

8. Saint-Girons H, Duguy R. 1963. Notes de citologie sanguine comparé sur les reptiles de France. B Soc Zool Fr 88: 613-624. 
9. Silveira C. 2006. Valores hematológicos de referência para papagaios-verdadeiros (Amazona aestiva - Psittacidae) mantidos em cativeiro. Tesis do Mestre. Univ do Belo Horizonte. $80 \mathrm{p}$.

10. Soto C, Acosta I, Cruz E. 2009. Parámetros hematológicos de Cotorras (Amazona leucocephala) y Cateyes (Aratinga euops). [Internet], [28 octubre 2010]. Rev Electron Vet 11(7B). Disponible en: http://www.veterinaria.org/revistas/ redvet/n070709B/070907.pdf

11. Soto C. 2010. Las psitacidas cubanas y su preservación. [Internet], [28 octubre
2010]. Rev Electron Vet 10(11B). Disponible en: http://www.veterinaria.org/ revistas/redvet/n111110B/111006B.pdf

12. Tell LA, Kabbur MB, Smith WL, Dahl KH, Cullor JS. 1997. A technique for isolating heterophils from blood of Orange-Winged Amazon Parrots (Amazona amazonica amazonica). Comp Haematol Int 7: 47-53.

13. Tobias J, Brightsmith D. 2007. Distribution, ecology and conservation status of the Blue-headed Macaw Primolius couloni. Biol Conserv 139: 126-138. 